\title{
Unbounded Jacobi Matrices with a Few Gaps in the Essential Spectrum: Constructive Examples
}

\author{
Anne Boutet de Monvel, Jan Janas and Serguei Naboko
}

\begin{abstract}
We give explicit examples of unbounded Jacobi operators with a few gaps in their essential spectrum. More precisely a class of Jacobi matrices whose absolutely continuous spectrum fills any finite number of bounded intervals is considered. Their point spectrum accumulates to $+\infty$ and $-\infty$. The asymptotics of large eigenvalues is also found.
\end{abstract}

Mathematics Subject Classification (2010). Primary 47B36; Secondary 47A10, 47B25.

Keywords. Jacobi matrix, essential spectrum, gaps, asymptotics.

\section{Introduction}

In this paper we look for examples of unbounded Jacobi matrices with several gaps in the essential spectrum. Let $\ell_{0}^{2}=\ell_{0}^{2}(\mathbb{N})$ be the space of sequences $\left\{f_{k}\right\}_{1}^{\infty}$ with a finite number of nonzero coordinates. For given real sequences $\left\{a_{k}\right\}_{1}^{\infty}$ and $\left\{b_{k}\right\}_{1}^{\infty}$ the Jacobi operator $\mathcal{J}_{0}$ acts in $\ell_{0}^{2}$ by the formula

$$
\left(\mathcal{J}_{0} f\right)_{k}=a_{k-1} f_{k-1}+b_{k} f_{k}+a_{k} f_{k+1}
$$

where $k=1,2, \ldots$ and $a_{0}=f_{0}=0$. The $a_{k}$ 's and $b_{k}$ 's are called the weights and diagonal terms, respectively.

In what follows we deal with positive sequences $\left\{a_{k}\right\}_{1}^{\infty}$. By Carleman's criterion [1] one can extend $\mathcal{J}_{0}$ to a self-adjoint operator $\mathcal{J}$ in $\ell^{2}=\ell^{2}(\mathbb{N})$ provided that

$$
\sum_{k} \frac{1}{a_{k}}=+\infty
$$

The above mentioned aim of finding an example of unbounded Jacobi operator $\mathcal{J}$ such that $\mathbb{R} \backslash \sigma_{\text {ess }}(\mathcal{J})$ consists of the union of several intervals could have been solved by using a general theorem of Stone. This theorem asserts that for a finite Borel measure $\mu$ on $\mathbb{R}$ one can find sequences $\left\{a_{k}\right\}_{1}^{\infty}$ and $\left\{b_{k}\right\}_{1}^{\infty}$ (expressed in terms of some moments of $\mu$ ) such that the operator of multiplication by $x$ in $L^{2}(\mu)$ is unitarily equivalent to the Jacobi operator 
associated to these sequences by (1.1) [23]. Therefore, one can easily prove the existence of unbounded Jacobi operators with arbitrary many gaps. However, we want to find explicit examples of $\left\{a_{k}\right\}_{1}^{\infty}$ and $\left\{b_{k}\right\}_{1}^{\infty}$ which define $\mathcal{J}$ with a few gaps in its essential spectrum.

The construction given below uses the Gilbert-Pearson subordination theory of Jacobi matrices [14] combined with the asymptotic analysis of formal solutions (generalized eigenvectors) of the system

$$
a_{n-1} u_{n-1}+b_{n} u_{n}+a_{n} u_{n+1}=\lambda u_{n}
$$

where $n=2,3, \ldots$ and $\lambda \in \mathbb{R}$. One can rewrite (1.2) in the standard form

$$
\vec{u}_{n+1}=B_{n}(\lambda) \vec{u}_{n}
$$

where $n=2,3, \ldots$ and

$$
\begin{gathered}
\vec{u}_{n}:=\left(\begin{array}{l}
u_{n-1} \\
u_{n}
\end{array}\right), \\
B_{n}(\lambda):=\left(\begin{array}{cc}
0 & 1 \\
-\frac{a_{n-1}}{a_{n}} & \frac{\lambda-b_{n}}{a_{n}}
\end{array}\right) .
\end{gathered}
$$

The matrix $B_{n}(\lambda)$ is called the "transfer matrix" and will be used below in our analysis of generalized eigenvectors.

It is well known that bounded Jacobi matrices with almost periodic entries have infinitely many gaps in the essential spectrum (in the generic case) [22]. We do not know any paper with similar results in the case of unbounded Jacobi matrices. However, we could expect that these results hold true for them. This is not our aim here because we want to keep control on the form of gaps in the essential spectrum in terms of some parameters which appear in the entries of Jacobi matrices we shall construct below. Moreover, this control might allow to construct examples of unbounded Jacobi matrices with a few so called "mobility edges". In the case of one gap such a construction has been done in our recent work [13]. Finally, we should mention a recent paper of Christiansen et al. [4] concerning finite gap bounded Jacobi matrices which describes the so called "isospectral torus".

Recall that to our best knowledge almost all explicit examples of unbounded Jacobi matrices considered in the last 30 years had essential spectra either empty or equal to the whole real line or a half line. Only several years ago appeared the first explicit examples of unbounded Jacobi matrices with one gap in the essential spectrum. These examples are given by the following expressions:

$$
\begin{aligned}
& a_{n}=n^{\alpha}+c_{n}, \\
& b_{n} \equiv 0,
\end{aligned}
$$

where $\alpha \in(0,1]$ and $\left\{c_{n}\right\}$ is a periodic sequencce of period two with $c_{1}-c_{2} \neq$ 0 . It turns out that the Jacobi operator $\mathcal{J}_{1}$ defined by these sequences has

$$
\sigma_{\text {ess }}\left(\mathcal{J}_{1}\right)=\sigma_{\text {ac }}\left(\mathcal{J}_{1}\right)=\left(-\infty,-\left|c_{1}-c_{2}\right|\right] \cup\left[\left|c_{1}-c_{2}\right|,+\infty\right),
$$

see $[6,8,12,17]$. Below we shall try to explain the origin of this phenomena from a general point of view. 
It turns out that a similar strategy (local periodicity) is also crucial for the formation of gaps in the essential spectrum of the unbounded Jacobi matrices considered in the present work. In particular the characteristic polynomial $p(\lambda)$ [see (3.1)] resemble but is different from the one of bounded Jacobi matrices. By choosing (locally) the entries $a_{n}$ as suitable powers of a small parameter $\varepsilon$, it is proved that the absolutely continuous spectrum $E$ of $J$ consists of exactly $N$ disjoint intervals. However, this $E$ is not the same as the corresponding one in the case of bounded periodic Jacobi matrices. In particular, the calculations in the last section show that we can construct examples with an arbitrary number of gaps in $\sigma_{\text {ess }}(J)$. Note that the essential spectrum of $J$ does not coincide with the absolutely continuous one of the corresponding periodic Jacobi matrix. Hence the results on the existence of gaps do not follow from the classical theory of periodic Jacobi matrices.

In this paper we concentrate on explicit examples of unbounded Jacobi matrices with finitely many (arbitrary many) intervals of absolutely continuous spectrum. However, in our examples the essential spectrum is bounded. The alternate case of finitely many bounded gaps in the essential spectrum will be considered in a separate paper by using a different technique.

The paper is organized as follows. In Sect. 2.1 we present the main idea of the construction in the simplest case of one gap, through analyzed in the above mentioned papers from an essentially different point of view. In Sect. 2.2 we describe the explicit construction of examples of unbounded Jacobi matrices with finitely many gaps. Its generalization via perturbation theory is considered in Sect. 2.3. The next Sect. 3 deals with the detailed analysis of the case of an arbitrary even "period" $N$ [see (2.1)]. Calculation of the absolutely continuous spectrum of the constructed Jacobi operator (for general even $N$ ) is given in Sect. 5. Moreover, the nondegeneracy of the gaps structure is shown there. Finally, the asymptotics of the discrete spectrum is under consideration in Sect. 4.

\section{Construction of Explicit Examples with Several Gaps}

\subsection{Heuristics}

The idea of the construction of an unbounded Jacobi matrix $\mathcal{J}$ with several gaps in $\sigma_{\text {ess }}(\mathcal{J})$ is based on the following heuristic reasoning.

Take a family of infinite Jacobi matrices (for simplicity all with zero diagonal, $\left.b_{n} \equiv 0\right)$ and periodic with respect to some parameters. The essential spectrum of any member of the family consists of a finite union of intervals which are symmetric with respect to zero

$$
\left(-x_{M},-y_{M}\right) \cup \cdots \cup\left(-x_{1},-y_{1}\right) \cup\left(y_{1}, x_{1}\right) \cup \cdots \cup\left(y_{M}, x_{M}\right),
$$

for an integer $M$. We choose some parameters of these Jacobi matrices in such a way that the "internal" intervals

$$
\left(-x_{M-1},-y_{M-1}\right), \ldots,\left(y_{M-1}, x_{M-1}\right)
$$

are almost fixed, and try to choose other parameters tending to infinity, with $\left(-x_{M},-y_{M}\right),\left(y_{M}, x_{M}\right)$, tending to $-\infty,+\infty$ respectively. Next we construct 
an unbounded Jacobi matrix $\mathcal{J}$ (with $b_{n} \equiv 0$ ) which looks similar (locally in index $n$ ) to the above ones from the family (but surely not a periodic one). Moreover, each copy of our periodic family appears "approximately" within the matrix $\mathcal{J}$ on some (eventually extended) sequence of matrix elements of index $n$. Additionally we shall try to choose the parameters smoothly or rather adiabatically to be able to track the asymptotics of solutions of (1.2) and therefore to control the a.c. spectrum. These heuristic arguments will be made precise below.

\section{The One Gap Case}

We illustrate the above idea in the one gap case, which was first found in [17]. However, the reasoning given in [17] was completely different. Let $\mathcal{J}_{\mathrm{p}}$ be the periodic Jacobi matrix given by $b_{n} \equiv 0$ and

$$
a_{2 n-1}=w_{1}, \quad a_{2 n}=w_{2}, \quad w_{1}>w_{2}>0 .
$$

Then the product $B_{2} B_{1}$ of the transfer matrices is

$$
B_{2} B_{1}=\left(\begin{array}{ll}
0 & 1 \\
\frac{w_{1}}{w_{2}} & \frac{\lambda}{w_{2}}
\end{array}\right)\left(\begin{array}{ll}
0 & 1 \\
\frac{w_{2}}{w_{1}} & \frac{\lambda}{w_{1}}
\end{array}\right)=\left(\begin{array}{ll}
-\frac{w_{2}}{w_{1}} & \frac{\lambda}{w_{1}} \\
-\frac{\lambda}{w_{1}} & -\frac{w_{1}}{w_{2}}+\frac{\lambda^{2}}{w_{1} w_{2}}
\end{array}\right) .
$$

Two bands of $\sigma_{\mathrm{ac}}\left(\mathcal{J}_{\mathrm{p}}\right)$ are given by

$$
\left\{\lambda|| \operatorname{tr}\left(B_{2} B_{1} \mid \leq 2\right\},\right.
$$

see [24]. It is easy to check that $\sigma_{\text {ac }}\left(\mathcal{J}_{\mathrm{p}}\right)$ is the union of two intervals

$$
\left[-\left(w_{1}+w_{2}\right),-\left(w_{1}-w_{2}\right)\right] \cup\left[w_{1}-w_{2}, w_{1}+w_{2}\right] .
$$

We may keep $w_{1}-w_{2}$ equal to a fixed positive constant $c$ but with $w_{1}+w_{2}$ tending to $+\infty$. For example we can choose

$$
w_{1}=n^{\alpha}+c_{1}, \quad \alpha \in(0,1], \quad w_{2}=n^{\alpha}+c_{2}, \quad c=\left|c_{1}-c_{2}\right| .
$$

In this way we obtain a 2-periodic perturbation of the Jacobi matrix with weights $a_{n}=n^{\alpha}$ and zero diagonal which was studied in $[5,6,8,12,17,21]$. Below we shall exploit a similar idea in the construction of our example.

\subsection{Basic Examples}

According to the above heuristics we define basic examples of Jacobi matrices as follows.

Definition $1(\mathcal{J}(\alpha))$. Let $z_{1}, \ldots, z_{N}$ be $N$ positive numbers and $\alpha \in(0,1]$. The Jacobi matrix $\mathcal{J}(\alpha)$ is defined by zero diagonal and weights

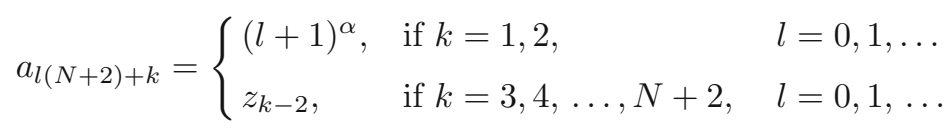

Remark. We must assume that all $z_{i} \neq 0$, otherwise $\mathcal{J}(\alpha)=\oplus M_{s}$, for some finite Jacobi matrices $M_{s}$.

One can prove that for $N=4$ the spectral picture of $\mathcal{J}(\alpha)$ has the following form (in the generic case) (Fig. 1). 


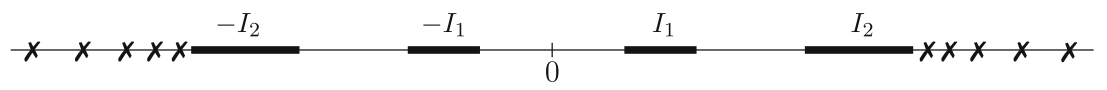

Figure 1. Spectrum of $\mathcal{J}(\alpha)$ for $N=4$

Here

$$
\sigma_{\text {ac }}(\mathcal{J}(\alpha))=-I_{2} \cup-I_{1} \cup I_{1} \cup I_{2}
$$

with known intervals $I_{1}, I_{2}$ and some eigenvalues of $\mathcal{J}(\alpha)$ denoted by $\times$ which tend to $\mp \infty$. This last assertion is obvious because $\mathcal{J}(\alpha)$ is an unbounded Jacobi matrix.

The above choice of the position of the weights $l^{\alpha}$ in the $l$-th block, $l=1,2, \ldots$ :

$$
\left(l^{\alpha}, l^{\alpha}, z_{1}, \ldots, z_{N}\right)
$$

could surely be different but definitively not arbitrary. For another example with the $l$-th block of weights given by

$$
\left(l^{\alpha}, z_{1}, \ldots, z_{N}, l^{\alpha}\right)
$$

we obtain a similar spectral picture. What is essential for both examples is that the norm of $B_{(N+2) l+3}(\lambda) B_{(N+2) l+2}(\lambda) B_{(N+2) l+1}(\lambda)$ remains bounded and smooth in $l$, as $l \rightarrow \infty$. However, $\left\|B_{(N+2) l+2}(\lambda) B_{(N+2) l+1}(\lambda)\right\| \rightarrow \infty$, as $l \rightarrow \infty$.

In Sect. 5 we shall prove that indeed the spectral picture of $\mathcal{J}(\alpha)$ (described above for $N=4$ ) looks similar for general $N$.

Theorem 2. If $\mathcal{J}(\alpha)$ is the Jacobi operator defined by (2.1), then

$$
\sigma_{\text {ac }}(\mathcal{J}(\alpha)) \supset \bar{E}
$$

where E, defined by (2.5), is a finite collection of intervals filled by purely absolutely continuous spectrum of local multiplicity one a.e. with respect to the Lebesgue measure.

Proof. It turns out that the spectral analysis of $\mathcal{J}(\alpha)$ can be based on the asymptotic behaviour of generalized eigenvectors corresponding to all $\lambda$ which do not belong to $\cup_{i=1}^{N} \partial I_{i}$, where $\partial I_{i}$ is the boundary of $I_{i}$. Due to the irregular behaviour (jumps) of products of transfer matrices within blocks we shall study the products over the whole blocks, see [10]. These products behave regularly with respect to the indices $s$ of blocks. Using (1.3) we have

$$
\vec{u}_{(N+2) s+1}=B_{(N+2) s}(\lambda) \cdots B_{(N+2)(s-1)+1}(\lambda) \vec{u}_{(N+2)(s-1)+1} .
$$

We want to find the asymptotic behaviour of $\vec{u}_{(N+2) s+1}$ as $s \rightarrow+\infty$. By definition of the weights in the $s$-th block we have

$$
\begin{aligned}
& B_{(N+2) s}(\lambda) \cdots B_{(N+2)(s-1)+1}(\lambda) \\
& =\underbrace{\left(\begin{array}{cc}
0 & 1 \\
-\frac{z_{N-1}}{z_{N}} & \frac{\lambda}{z_{N}}
\end{array}\right) \cdots\left(\begin{array}{cc}
0 & 1 \\
-\frac{z_{1}}{z_{2}} & \frac{\lambda}{z_{2}}
\end{array}\right)}_{M(\lambda)} \underbrace{\left(\begin{array}{cc}
0 & 1 \\
-\frac{s^{\alpha}}{z_{1}} & \frac{\lambda}{z_{1}}
\end{array}\right)\left(\begin{array}{cc}
0 & 1 \\
-1 & \frac{\lambda}{s^{\alpha}}
\end{array}\right)\left(\begin{array}{cc}
0 & 1 \\
-\frac{z_{N}}{s^{\alpha}} & \frac{\lambda}{s^{\alpha}}
\end{array}\right)}_{C} .
\end{aligned}
$$


The product of the last three matrices above is

$$
C=\left(\begin{array}{ll}
-\frac{\lambda z_{N}}{s^{2 \alpha}} & -1+\frac{\lambda^{2}}{s^{2 \alpha}} \\
\frac{z_{N}}{z_{1}}-\frac{\lambda^{2} z_{N}}{z_{1} s^{2 \alpha}} & -\frac{2 \lambda}{z_{1}}+\frac{\lambda^{3}}{z_{1} s^{2 \alpha}}
\end{array}\right) .
$$

If $M(\lambda)$ denotes the product of the first $(N-1)$ matrices to the left of $C$, then we can rewrite the left hand side of (2.3) as

$$
A(\lambda)+s^{-2 \alpha} B(\lambda)
$$

where

$$
\begin{aligned}
& A(\lambda):=M(\lambda)\left(\begin{array}{cc}
0 & -1 \\
\frac{z_{N}}{z_{1}} & -\frac{2 \lambda}{z_{1}}
\end{array}\right), \\
& B(\lambda):=M(\lambda)\left(\begin{array}{cc}
-\lambda z_{N} & \lambda^{2} \\
-\frac{\lambda^{2} z_{N}}{z_{1}} & \frac{\lambda^{3}}{z_{1}}
\end{array}\right) .
\end{aligned}
$$

Note that $A(\lambda), B(\lambda)$ does not depend on $s$ and $B(\lambda)$ is not the monodromy matrix generated by the periodic sequence $z_{1}, \ldots, z_{N}$.

Next we observe that

$$
\operatorname{det}\left[A(\lambda)+s^{-2 \alpha} B(\lambda)\right]=\frac{z_{N-1}}{z_{N}} \ldots \frac{z_{1}}{z_{2}} \cdot \frac{s^{\alpha}}{z_{1}} \cdot \frac{z_{N}}{s^{\alpha}}=1 .
$$

If $\operatorname{disc} A:=(\operatorname{tr} A)^{2}-4 \operatorname{det} A$ denotes the discriminant of a $2 \times 2$ matrix $A$, then

$$
\operatorname{disc}\left[A(\lambda)+s^{-2 \alpha} B(\lambda)\right]=\left(\operatorname{tr}\left[A(\lambda)+s^{-2 \alpha} B(\lambda)\right]\right)^{2}-4 .
$$

Notation (elliptic part). We denote

$$
E:=\{\lambda \in \mathbb{R}|| \operatorname{tr} A(\lambda) \mid<2\} .
$$

Below we shall describe some properties of the function $\lambda \mapsto \operatorname{tr} A(\lambda)$. Now we only note that $\operatorname{tr} A(\lambda)$ is a polynomial in $\lambda$ of degree $N$. Therefore, each of the equations $\operatorname{tr} A(\lambda)= \pm 2$ has at most $N$ solutions. It follows that $E$ consists of at most $N$ intervals.

If $\lambda \in E$ then, for some $s_{0}$,

$$
\operatorname{disc}\left[A(\lambda)+s^{-2 \alpha} B(\lambda)\right]<0 \text { for any } s \geq s_{0} .
$$

Consequently, the product in (2.3) is an elliptic matrix for any $s \geq s_{0}$. Thus, the matrix $A(\lambda)+s^{-2 \alpha} B(\lambda)$ has two distinct eigenvalues

$$
\mu_{\lambda}^{(1)}(s), \mu_{\lambda}^{(2)}(s) \text { for } s \gg 1
$$

with

$$
\left|\mu_{\lambda}^{(i)}(s)\right|=1, \quad i=1,2 \text { and } \mu_{\lambda}^{(2)}(s)=\overline{\mu_{\lambda}^{(1)}(s)} .
$$

Consider the system of equations

$$
\vec{y}_{s}=\left(A(\lambda)+s^{-2 \alpha} B(\lambda)\right) \vec{y}_{s-1}, \quad \vec{y}_{s}:=\vec{u}_{(N+2) s+1}, \quad s=2,3, \ldots
$$


The above properties of the matrices $A(\lambda)+s^{-2 \alpha} B(\lambda)$ allow to apply [19, Theorem 3.2] and to obtain a basis of solutions of (1.3) with the following asymptotic formula

$$
\vec{u}_{(N+2) s}^{(k)}(\lambda)=\prod_{r=2}^{s-1} \mu_{\lambda}^{(k)}(r)\left(f_{k}+\mathrm{o}(1)\right), \quad k=1,2,
$$

where $f_{k}$ are the eigenvectors of $A(\lambda)$. By definition of the weights and by direct computation we also obtain the asymptotic behaviour of bases of solutions of (1.3) for the remaining subsequences of indices:

$$
\begin{aligned}
\vec{u}_{(N+2) s+1}^{(k)}(\lambda)= & B_{(N+2) s}(\lambda) \vec{u}_{(N+2) s}^{(k)}(\lambda), \\
\vec{u}_{(N+2) s+2}^{(k)}(\lambda)= & {\left[\left(\begin{array}{cc}
-\frac{z_{N-1}}{z_{N}} & \frac{\lambda}{z_{N}} \\
0 & 0
\end{array}\right)+(s+1)^{-\alpha}\left(\begin{array}{cc}
0 & 0 \\
-\frac{z_{N-1}}{z_{N}} & -z_{N}+\frac{\lambda^{2}}{z_{N}}
\end{array}\right)\right] } \\
& \times \vec{u}_{(N+2) s}^{(k)}(\lambda), \\
\vec{u}_{(N+2) s+3}^{(k)}(\lambda)= & \left(\begin{array}{cc}
0 & 1 \\
-1 & \lambda(s+1)^{-\alpha}
\end{array}\right) \vec{u}_{(N+2) s+2}^{(k)}(\lambda), \\
\vec{u}_{(N+2) s+4}^{(k)}(\lambda)= & {\left.\left[\begin{array}{cc}
0 & -1 \\
\frac{z_{N}}{z_{1}} & -\frac{2 \lambda}{z_{1}}
\end{array}\right)+(s+1)^{-2 \alpha}\left(\begin{array}{ll}
-\lambda z_{N} & \lambda^{2} \\
-\lambda^{2} z_{N} & \frac{\lambda^{3}}{z_{1}}
\end{array}\right)\right] } \\
\times \vec{u}_{(N+2) s+1}^{(k)}(\lambda), & \\
\vec{u}_{(N+2) s+l}^{(k)}(\lambda)= & \prod_{i=2}^{l-3} A_{i}(\lambda) \vec{u}_{(N+2) s+4}^{(k)}(\lambda), \quad l=5, \ldots, N+3,
\end{aligned}
$$

where

$$
A_{i}(\lambda)=\left(\begin{array}{ll}
0 & 1 \\
-\frac{z_{i-1}}{z_{i}} & \frac{\lambda}{z_{i}}
\end{array}\right) .
$$

Combining (2.6), (2.7), (2.8), (2.9), (2.10) and (2.11) we see that the system (1.2) has no subordinated solutions for $\lambda \in E[$ see (2.5)]. This completes the proof.

Notation (hyperbolic part). Now we turn to the remaining case of

$$
S:=\{\lambda \in \mathbb{R}|| \operatorname{tr} A(\lambda) \mid>2\} .
$$

It happens that in this case we again can find the asymptotic behaviour of generalized eigenvectors for $\lambda \in S$. This hyperbolic asymptotics will allow us to prove that the spectrum of $\mathcal{J}(\alpha)$ in $S$ is pure point. In order to prove its discreteness we assume that $\alpha \in\left(\frac{1}{2}, 1\right)$. This restriction is not necessary. The results (we prove below) hold true for any $\alpha \in(0,1]$ but their proofs are less elementary.

Theorem 3. The spectrum of $\mathcal{J}(\alpha)$ in $S$ is discrete. 
Proof. For $\vec{y}_{s}:=\vec{u}_{(N+2) s+1}$ we consider the system

$$
\vec{y}_{s}=\left(A(\lambda)+s^{-2 \alpha} B(\lambda)\right) \vec{y}_{s-1}, \quad \lambda \in S, \quad s=2,3, \ldots
$$

By diagonalization of $A(\lambda)$ we can write $A(\lambda)=V(\lambda) D(\lambda) V(\lambda)^{-1}$ with $D(\lambda)$ diagonal. Hence, for $\vec{z}_{s}:=V(\lambda)^{-1} \vec{y}_{s}$ we have the new system

$$
\vec{z}_{s}=\left(D(\lambda)+s^{-2 \alpha} B_{1}(\lambda)\right) \vec{z}_{s-1}, \quad s=2,3, \ldots
$$

where $B_{1}(\lambda)=V^{-1}(\lambda) B(\lambda) V(\lambda)$. All functions $V(\lambda), V(\lambda)^{-1}, D(\lambda)$ of $\lambda$ can be chosen continuous [8].

Fix a bounded interval $I \subset S$. Since $\alpha>\frac{1}{2}$ we can apply a uniform version of the Benzaid-Lutz result [19, Theorem 3.2] and obtain a basis $\vec{\phi}_{n}^{(k)}(\lambda)$ $(k=1,2)$ of solutions of (2.13) such that

$$
\sup _{\lambda \in I}\left\|\vec{\phi}_{n}^{(k)}(\lambda)\left(\prod_{i=N_{0}}^{n-1} \mu_{i}^{(k)}(\lambda)\right)^{-1}-e_{k}\right\| \underset{n \rightarrow+\infty}{\stackrel{\longrightarrow}{\longrightarrow}} 0
$$

the spectrum

$$
\sigma\left(A(\lambda)+s^{-2 \alpha} B(\lambda)\right)=\left\{\mu_{s}^{(1)}(\lambda), \mu_{s}^{(2)}(\lambda)\right\}
$$

and $N_{0}$ is so large that $\operatorname{tr}\left(A(\lambda)+s^{-2 \alpha} B(\lambda)\right)<-2$ for all $s \geq N_{0}$. The case $\operatorname{tr}\left(A(\lambda)+s^{-2 \alpha} B(\lambda)\right)>2$ is similar. It follows that

$$
0<\mu_{s}^{(1)}(\lambda):=\frac{1}{2}\left(-\operatorname{tr} A_{s}-\sqrt{\operatorname{disc} A_{s}}\right)<1,
$$

where $A_{s}:=A(\lambda)+s^{-2 \alpha} B(\lambda)$. Using (2.14) and (2.15) we have

$$
\left\{\sup _{\lambda \in I}\left\|\vec{\phi}_{n}^{(1)}(\lambda)\right\|\right\}_{n=1}^{\infty} \in \ell^{2} .
$$

This can be easily seen, by repeating the reasoning given in the proofs of $(2.7),(2.8),(2.9),(2.10)$ and (2.11). In particular, the "eigenvalue equation" (1.2) has a nontrivial subordinated solution.

Consequently, $S \cap \sigma_{\mathrm{ac}}(\mathcal{J}(\alpha))=\varnothing$ and so $E \subset \sigma_{\mathrm{ac}}(\mathcal{J}(\alpha)) \subset \bar{E}$ (by Theorem 2). The subordination theory shows that $\sigma_{\mathrm{sc}}(\mathcal{J}(\alpha))$ is empty [15]. Therefore, $S$ contains only eigenvalues of $\mathcal{J}(\alpha)$. Using [19, Lemma 4.2] for a given $n \geq N_{0}$ and any $\varepsilon>0$ one can find $\delta>0$ such that

$$
\left.\begin{array}{l}
|\lambda-\mu|<\delta \\
\lambda, \mu \in I
\end{array}\right\} \Longrightarrow\left\|\vec{\phi}_{n}^{(1)}(\lambda)-\vec{\phi}_{n}^{(1)}(\mu)\right\|<\varepsilon .
$$

Now we can repeat the argument of the proof of [19, Theorem 5.3]. Although this argument was given for a particular class of Jacobi matrices it can be applied in general situations. To prove discreteness of $\sigma(\mathcal{J}(\alpha))$ in $S$ assume that there exists a sequence of distinct eigenvalues $\left\{\varrho_{m}\right\}$ of $\mathcal{J}(\alpha)$ which accumulates at $\varrho \in S$. Altogether (2.14) provides a uniform tails estimate, and (2.16) guarantees the coordinate convergence

$$
\vec{\phi}_{n}^{(1)}\left(\varrho_{m}\right) \underset{m \rightarrow \infty}{\longrightarrow} \vec{\phi}_{n}^{(1)}(\varrho) .
$$

This allows to repeat the reasoning given in [19, p. 184]. 
Indeed, by orthogonality of the eigenvectors $\left\{\vec{\phi}_{n}^{(1)}\left(\varrho_{s}\right)\right\}$ of $\mathcal{J}(\alpha)$ (for different $\varrho_{s}$ ) we have

$$
0=\lim _{\substack{m \rightarrow \infty \\ k \rightarrow \infty \\ k \neq m}}\left\langle\left\{\vec{\phi}_{n}^{(1)}\left(\varrho_{k}\right)\right\},\left\{\vec{\phi}_{n}^{(1)}\left(\varrho_{m}\right)\right\}\right\rangle_{\ell^{2}}=\sum_{s=1}^{+\infty}\left|\vec{\phi}_{s}^{(1)}(\varrho)\right|^{2} .
$$

The last equality can be easily verified by using (2.14) and (2.16). Therefore, $\vec{\phi}_{n}^{(1)}(\varrho)=0$, and this contradiction completes the proof of Theorem 3$)$.

\subsection{Generalization}

Slight perturbations of the above examples allow to obtain a class of unbounded Jacobi matrices with the same spectral picture as before.

Notation. $D^{1}$ denotes the space of bounded variation sequences.

Definition $4(\widetilde{\mathcal{J}}(\alpha))$. The Jacobi matrix $\widetilde{\mathcal{J}}(\alpha)$ is defined by zero diagonal and by the new weights

$$
\tilde{a}_{l(N+2)+k}= \begin{cases}(l+1)^{\alpha}+c_{k}(l), & \text { if } k=1,2, \quad l=0,1, \ldots \\ z_{k-2}+t_{k}(l), & \text { if } k=3, \ldots, N+2, \quad l=0,1, \ldots\end{cases}
$$

submitted to the following two assumptions:

$$
\begin{aligned}
& \left\{t_{k}(l)\right\}_{l \geq 0} \in \ell^{2} \cap D^{1}, \quad k=3,4, \ldots, N+2 . \\
& \left\{c_{1}(l) / l^{\alpha}\right\}_{l \geq 0} \&\left\{c_{2}(l) / l^{\alpha}\right\}_{l \geq 0} \in \ell^{2} \cap D^{1} .
\end{aligned}
$$

Remark. Note that (2.19) allows unbounded sequences $\left\{c_{1}(l)\right\}$ and $\left\{c_{2}(l)\right\}$.

By repeating the computations leading to (2.4) we find

$$
\begin{aligned}
& B_{(N+2) s}(\lambda) \cdots B_{(N+2)(s-1)+1}(\lambda) \\
&=\left(\begin{array}{ll}
0 & 1 \\
-\frac{z_{N-1}+t_{N-1}(l)}{z_{N}+t_{N}(l)} & \frac{\lambda}{z_{N}+t_{N}(l)}
\end{array}\right) \cdots\left(\begin{array}{ll}
0 & 1 \\
-\frac{z_{1}+t_{1}(l)}{z_{2}+t_{2}(l)} & \frac{\lambda}{z_{2}+t_{2}(l)}
\end{array}\right) \\
& \times\left(\begin{array}{ccc}
0 & 1 & \\
-\frac{l^{\alpha}+c_{2}(l)}{z_{1}+t_{1}(l)} & \frac{\lambda}{z_{1}+t_{1}(l)}
\end{array}\right)\left(\begin{array}{ll}
0 & 1 \\
-\frac{l^{\alpha}+c_{1}(l)}{l^{\alpha}+c_{2}(l)} & \frac{\lambda}{l^{\alpha}+c_{2}(l)}
\end{array}\right)\left(\begin{array}{ll}
0 \\
-\frac{z_{N}+t_{N}(l)}{l^{\alpha}+c_{1}(l)} & \frac{\lambda}{l^{\alpha}+c_{1}(l)}
\end{array}\right) .
\end{aligned}
$$

Note that due to (2.18) we have the following decomposition

$$
\left(\begin{array}{ll}
0 & 1 \\
-\frac{z_{s-1}+t_{s-1}(l)}{z_{s}+t_{s}(l)} & \frac{\lambda}{z_{s}+t_{s}(l)}
\end{array}\right)=C_{s}+W_{s l}(\lambda)+R_{s l}(\lambda),
$$

where

$$
\begin{aligned}
& C_{s}:=\left(\begin{array}{ll}
0 & 1 \\
-\frac{z_{s-1}}{z_{s}} & \frac{\lambda}{z_{s}}
\end{array}\right), \\
& W_{s l}(\lambda):=\left(\begin{array}{cc}
0 & 0 \\
\frac{z_{s-1} t_{s}(l)-z_{s} t_{s-1}(l)}{z_{s}{ }^{2}} & -\frac{\lambda t_{s}(l)}{z_{s}}
\end{array}\right), \\
& \left\{\left\|R_{s l}(\lambda)\right\|\right\}_{l \geq 0} \in \ell^{1}, \quad s=2, \ldots, N .
\end{aligned}
$$


Using (2.21) and distributive rule in (2.20) we can rewrite the product of the first $N-1$ matrices of the right side of (2.20) as follows:

$$
M(\lambda)+\sum_{r=2}^{N-1} C_{N} \ldots C_{r+1} W_{r l}(\lambda) C_{r-1} \ldots C_{2}+R_{l}^{(1)}(\lambda),
$$

with $\left\{\left\|R_{l}^{(1)}(\lambda)\right\|\right\}_{l \geq 0} \in \ell^{1}$. Since $\left\{W_{r l}(\lambda)\right\}_{l \geq 0} \in \ell^{2} \cap D^{1}$ the above product has the form:

$$
M(\lambda)+T_{l}(\lambda)+R_{l}^{(1)}(\lambda)
$$

where $\left\{T_{l}(\lambda)\right\}_{l \geq 0} \in \ell^{2} \cap D^{1}$.

In turn, due to (2.19) the product of the three last matrices of the right side of $(2.20)$ can be written

$$
D_{N}+V_{l}+R_{l}^{(2)}(\lambda)
$$

with

$$
\begin{aligned}
& D_{N}=\left(\begin{array}{ll}
0 & -1 \\
\frac{z_{N}}{z_{1}} & -2 \frac{\lambda}{z_{1}}
\end{array}\right) \\
& V_{l}=\left(\begin{array}{ll}
0 & \left(c_{2}(l)-c_{1}(l)\right) l^{-\alpha} \\
\frac{z_{1}^{2} t_{N}(l-1)-z_{N} t_{N}(l)}{z_{1}{ }^{2}}+\frac{z_{N}\left(c_{2}(l)-c_{1}(l)\right.}{z_{1} l^{-\alpha}} & 0
\end{array}\right), \\
& \left\{\left\|R_{l}^{(2)}(\lambda)\right\|\right\}_{l \geq 0} \in \ell^{1} .
\end{aligned}
$$

Combining (2.22) and (2.23) we rewrite the right hand side of (2.20) as

$$
A(\lambda)+S_{l}(\lambda)+R_{l}^{(3)}(\lambda)
$$

where

$$
\begin{aligned}
& A(\lambda)=M(\lambda) D_{N}(\lambda), \\
& \left\{S_{l}(\lambda)\right\}_{l \geq 0} \in \ell^{2} \cap D^{1}, \\
& \left\{\left\|R_{l}^{(3)}(\lambda)\right\|\right\}_{l \geq 0} \in \ell^{1} .
\end{aligned}
$$

By repeating the proofs of Theorems 2 and 3 we obtain

Theorem 5. Let $\tilde{J}(\alpha)$ be the Jacobi operator defined by the weights (2.17) submitted to conditions (2.18) and (2.19) with $\alpha>1 / 2$. Then the statements of Theorems 2 and 3 remain valid for $\tilde{J}(\alpha)$.

Proof (sketch). We first note that due to the definitions of $R_{s l}(\lambda), T_{l}(\lambda), M(\lambda)$ and (2.24) the error terms $R_{l}^{(1)}(\lambda)$ and $R_{l}^{(2)}(\lambda)$ are continuous functions of $\lambda$. Moreover, both $R_{l}^{(1)}(\lambda)$ and $R_{l}^{(2)}(\lambda)$ have uniform tail estimates, i.e. for any compact subset $X \subset \mathbb{R}$ and for any $\varepsilon>0$ there exists $K=K(\varepsilon, X)$ such that

$$
\sup _{\lambda \in X} \sum_{l=K}^{\infty}\left\|R_{l}^{(s)}(\lambda)\right\|<\varepsilon, \quad s=1,2 .
$$


Consequently, $R_{l}^{(3)}(\lambda)$ satisfies similar tail estimates. Remember that $A(\lambda)$ and $S_{l}(\lambda)$ are also continuous functions of $\lambda$ (to be more precise they are polynomials in $\lambda$ ).

Using a uniform Levinson type theorem (see [19] for the case of uniform $\ell^{1}$ perturbations and [20] for the case of uniform $D^{1}$ ones) one can easily complete the proof of the analogues of Theorems 2 and 3.

Remark. By adding a nonzero main diagonal $\left\{b_{k}\right\} \in \ell^{2} \cap D^{1}$ one obtains a still larger class of Jacobi matrices with the same spectral picture as $\mathcal{J}(\alpha)$. This surely introduces a compact perturbation of the Jacobi operator and by Weyl's theorem it preserves the essential spectrum, but our goal is to preserve $\sigma_{\text {ac }}(\mathcal{J}(\alpha))$.

\section{Analysis of Particular Examples}

One of the aims of particular examples analysis is to verify the nondegeneracy of arbitrary many gaps in the essential spectrum.

\section{Properties of the Characteristic Polynomial}

We shall discuss some properties of the polynomial $p(\lambda):=\operatorname{tr} A(\lambda)$ which will be useful below.

We define the antidiagonal and diagonal matrices, respectively

$$
A_{k}:=\left(\begin{array}{ll}
0 & 1 \\
-\frac{z_{k-1}}{z_{k}} & 0
\end{array}\right), \quad B_{k}:=\left(\begin{array}{cc}
0 & 0 \\
0 & \frac{1}{z_{k}}
\end{array}\right), \quad k=1, \ldots, N
$$

where $z_{0}:=z_{N}$. In what follows we assume that $N$ is an even number and all $z_{j}$ are positive (without loss of generality). We have

$$
p(\lambda)=\operatorname{tr}\left[\left(A_{N}+\lambda B_{N}\right) \cdots\left(A_{2}+\lambda B_{2}\right)\left(-A_{1}-2 \lambda B_{1}\right)\right]=: \sum_{k=0}^{N} c_{k} \lambda^{k} .
$$

Lemma 3.1. Let $N$ be an even number. Then the coefficients $c_{k}$ of the characteristic polynomial $p(\lambda):=\operatorname{tr} A(\lambda)$ satisfy:

(i) $c_{2 m+1}=0$, for $m=0,1,2, \ldots$

(ii) The sign of $c_{2 m}$ coincides with $-(-1)^{\frac{N-2 m}{2}}$.

Proof. We pick up the first $n_{1}$ antidiagonal terms $A_{i}\left(i=N, \ldots, N-n_{1}+1\right)$. After that in the next to the right matrix we take only the term $\lambda B_{N-n_{1}}$. The same procedure can be repeated, i.e., we pick up the next $n_{2}$ antidiagonal matrices $A_{s}\left(s=N-n_{1}-1, \ldots, N-n_{1}-n_{2}\right)$, etc.

Totally, we get a sequence $n_{1}, n_{2}, \ldots, n_{k+1}, 0 \leq n_{s} \leq N$ such that $\sum_{s=1}^{k+1} n_{s}=N-k$. Observe that all the traces

$$
\operatorname{tr}\left(A_{N} \cdots A_{N-n_{1}+1} B_{N-n_{1}} \cdots\right)=0,
$$

provided that $k$ is odd. This is clear because then $N-k$ is an odd number and therefore the above product $A_{N} \cdots A_{N-n_{1}+1} B_{N-n_{1}} \cdots$ has an odd number of anti-diagonal matrices $A_{s}$ and a certain number of diagonal matrices $B_{r}$. Hence the whole product gives an anti-diagonal matrix (therefore with zero 
trace). Moreover, in all nonzero terms $\operatorname{tr}\left(A_{N} \cdots A_{N-n_{1}+1} B_{N-n_{1}} \cdots\right)$ all the numbers $n_{1}, n_{2}, \ldots, n_{k+1}$ must be even. Indeed, this fact is a consequence of the following elementary matrix algebra. First note that due to the invariance of the trace with respect to the cyclic permutations one can reduce the proof to the case that $n_{1}$ should be even. Assume for a moment that $n_{1}$ is an odd number. Then the first to the left product of $n_{1}$ anti-diagonal matrices $A_{N} \cdots A_{N-n_{1}+1}$ is again an anti-diagonal matrix. The next term $B_{N-n_{1}}$ has the form $\left(\begin{array}{cc}0 & 0 \\ 0 & \star\end{array}\right)$ with one nonzero entry in the right lower corner. The remaining product of matrices contains $k-1$ diagonal matrices $B_{s}$ and $N-n_{1}-k$ anti-diagonal matrices $A_{s}$. Since the numbers $N$ and $k$ are even and $n_{1}$ is odd, so $N-n_{1}-k$ is odd, and therefore the remaining product must also contain an odd number $n_{r}$ of matrices $A_{i}$ 's. We can assume that the remaining product begins from the left with an anti-diagonal matrix. In fact, all diagonal factors at the left of the remaining product multiplied by $B_{N-n_{1}}$ would produce a matrix of the form $\left(\begin{array}{ll}0 & 0 \\ 0 & \star\end{array}\right)$. Therefore, the whole product would have the following structure

$$
\left(\begin{array}{cc}
0 & \star \\
\star & 0
\end{array}\right)\left(\begin{array}{cc}
0 & 0 \\
0 & \star
\end{array}\right)\left(\begin{array}{ll}
0 & \star \\
\star & 0
\end{array}\right)\left(\begin{array}{ll}
* & \\
& *
\end{array}\right) \cdots\left(\begin{array}{ll}
* & \\
& *
\end{array}\right)
$$

where all matrices $\left(\begin{array}{ll}* & \\ & *\end{array}\right)$ are diagonal. But the product of these diagonal matrices $\left(\begin{array}{ll}* & \\ & *\end{array}\right)$ contains $B_{i}$ 's and so the product of all of them has the form $\left(\begin{array}{cc}0 & 0 \\ 0 & \star\end{array}\right)$. It follows that the whole product is equal to

$$
\left(\begin{array}{ll}
0 & \star \\
\star & 0
\end{array}\right)\left(\begin{array}{ll}
0 & 0 \\
0 & \star
\end{array}\right)\left(\begin{array}{ll}
0 & \star \\
\star & 0
\end{array}\right)\left(\begin{array}{ll}
0 & 0 \\
0 & \star
\end{array}\right)=\left(\begin{array}{ll}
0 & 0 \\
0 & 0
\end{array}\right)
$$

This contradiction proves that $n_{1}$ is even. A more detailed analysis of the product of $n_{i}$ anti-diagonal $A_{s}$ shows that it is a positive diagonal matrix muliplied by $(-1)^{\frac{n_{i}}{2}}$. This fact can be easily checked using the structure of

$$
A_{s}=\left(\begin{array}{ll}
0 & \alpha_{s} \\
\beta_{s} & 0
\end{array}\right), \quad \alpha_{s}>0, \beta_{s}<0
$$

Since all $B_{s}$ have non-negative entries, the sign of $c_{k}$ (for even $k$ ) coincides with

$$
-(-1)^{\frac{n_{1}}{2}+\cdots+\frac{n_{k}}{2}}=-(-1)^{\frac{N-k}{2}} .
$$

Therefore, the signs of $c_{0}, c_{2}, \ldots, c_{N}$ form an alternating sequence. In particular, the sign of $c_{0}$ is equal to $-(-1)^{\frac{N}{2}}$. The proof is complete.

Remark 3.2. Note that vanishing of $c_{2 m+1}$ is not surprising, due to the symmetry $\lambda \mapsto-\lambda$ of $\sigma(\mathcal{J}(\alpha))$ with vanishing main diagonal of $\mathcal{J}(\alpha)$. 
Remark 3.3. Let $N=2 M$. Denote $\mu:=\lambda^{2}$. Then

$$
P(\mu):=p(\sqrt{\mu})=c_{2 M} \mu^{M}+\cdots+c_{2} \mu+c_{0},
$$

with alternating signs of the coefficients. Observe that

$$
c_{2 M}=-\frac{2}{z_{N} z_{N-1} \ldots z_{1}}
$$

and so $c_{2 M} \neq 0$.

\section{Asymptotics of the Discrete Spectrum at Infinity}

In this section we shall find asymptotic formulae for the eigenvalues of $\mathcal{J}(\alpha)$ at $\pm \infty$. More precisely, we shall compute the main terms of these formulae (modulo bounded corrections). In recent years appeared several papers devoted to the asymptotic behaviour of the eigenvalues of unbounded, selfadjoint Jacobi matrices $[3,7,11,16,25]$. However, the results found in these works do no apply to $\mathcal{J}(\alpha)$. Therefore, we have decided to include asymptotic formulas for the eigenvalues of $\mathcal{J}(\alpha)$, although they are not the main concern of this work.

The idea of computing approximate values of $\sigma_{\mathrm{p}}(\mathcal{J}(\alpha))$ (for sufficiently large eigenvalues) is based on the following decomposition

$$
\mathcal{J}(\alpha)=\mathcal{J}_{1}+\mathcal{J}_{2}
$$

where $\mathcal{J}_{1}, \mathcal{J}_{2}$ are Jacobi matrices and the weights of $\mathcal{J}_{1}$ are obtained from the weights of $\mathcal{J}(\alpha)$ by replacing all $z_{i}(i=1, \ldots, N)$ by zeros whereas the weights of $\mathcal{J}_{2}$ are defined by replacing all terms $\left(k^{\alpha}, k^{\alpha}\right)(k=1,2, \ldots)$ by zeros. The definition of $\mathcal{J}_{1}$ implies that

$$
\mathcal{J}_{1}=\bigoplus_{k=1}^{\infty}\left(M_{k} \oplus 0_{N}\right)
$$

where $0_{N}$ is the $(N-1) \times(N-1)$ zero matrix and

$$
M_{k}:=\left(\begin{array}{lll}
0 & k^{\alpha} & 0 \\
k^{\alpha} & 0 & k^{\alpha} \\
0 & k^{\alpha} & 0
\end{array}\right) .
$$

By definition of $\mathcal{J}_{2}$ we have

$$
\mathcal{J}_{2}=\bigoplus_{k=1}^{\infty}\left(0_{2} \oplus L_{k}\right),
$$


where

$$
L_{k}:=L:=\left(\begin{array}{ccccccc}
0 & z_{1} & 0 & 0 & \ldots & \ldots & 0 \\
z_{1} & 0 & z_{2} & 0 & \ldots & \ldots & 0 \\
0 & z_{2} & 0 & z_{3} & \ldots & \ldots & 0 \\
\vdots & \ddots & \ddots & \ddots & \ddots & \ddots & \vdots \\
\ldots & \ldots & \ldots & \ldots & \ldots & \ldots & 0 \\
\ldots & \ldots & \ldots & \ldots & z_{N-1} & 0 & z_{N} \\
0 & 0 & \ldots & \ldots & 0 & z_{N} & 0
\end{array}\right)
$$

Well known elementary estimates and (4.2) show that

$$
\left\|\mathcal{J}_{2}\right\| \leq 2 \max _{i}\left|z_{i}\right| \cdot \cos \frac{\pi}{N+1}
$$

and

$$
\sigma_{\text {ess }}\left(\mathcal{J}_{2}\right)=\{0\} \cup \sigma(L) .
$$

Applying [2, Theorem 4, Chap. 9, Sec. 4, p. 219] we have

$$
\sigma_{\text {ess }}(\mathcal{J}(\alpha)) \subset\left[-\left\|\mathcal{J}_{2}\right\|,\left\|\mathcal{J}_{2}\right\|\right]=[-\|L\|,\|L\|] .
$$

In what follows we shall consider $\sigma_{\mathrm{p}}(\mathcal{J}(\alpha))$ outside the interval $[-\|L\|,\|L\|]$. Since the spectrum of $\mathcal{J}(\alpha)$ outside this interval is discrete and symmetric with respect to zero $\left(b_{n} \equiv 0\right)$ it accumulates at $\mp \infty$.

Denote $\|L\|=a$, and take a large number $M \gg a$. For a self-adjoint operator $T$, let $\Pi_{T}(\alpha, \beta)$ be the number of eigenvalues of $T$ in the interval $(\alpha, \beta)$.

At this point we use standard arguments of perturbation theory. Namely applying [2, Lemma 3, Chap. 9, Sec. 4, p. 218] twice (with $A=\mathcal{J}_{1}, V=\mathcal{J}_{2}$ or $A=\mathcal{J}(\alpha), V=-\mathcal{J}_{2}$, respectively) we have the inequalities

$$
\Pi_{\mathcal{J}_{1}}(2 a, M) \leq \Pi_{\mathcal{J}(\alpha)}(a, M+a) \leq \Pi_{\mathcal{J}_{1}}(0, M+2 a) .
$$

Note that

$$
\sigma\left(M_{k}\right)=\left\{-\sqrt{2} k^{\alpha}, 0, \sqrt{2} k^{\alpha}\right\} .
$$

By definition of $\mathcal{J}_{1}$ and standard arguments we can write

$$
\begin{gathered}
\Pi_{\mathcal{J}_{1}}(2 a, M) \sim\left(\frac{M}{\sqrt{2}}\right)^{\frac{1}{\alpha}}, \\
\Pi_{\mathcal{J}_{1}}(a, M+a) \sim\left(\frac{M}{\sqrt{2}}\right)^{\frac{1}{\alpha}}+\left(\frac{M}{\sqrt{2}}\right)^{\frac{1}{\alpha}} \frac{a}{\alpha M},
\end{gathered}
$$

as $M \rightarrow+\infty$. Combining (4.4), (4.5) and (4.6) we have

$$
\Pi_{\mathcal{J}(\alpha)}(a, M+a)=\left(\frac{M}{\sqrt{2}}\right)^{\frac{1}{\alpha}}+r_{M},
$$

where $r_{M}=\mathrm{O}\left(M^{\frac{1}{\alpha}-1}\right)$, as $M \rightarrow+\infty$. Let

$$
1+a<\lambda_{1}(\mathcal{J}(\alpha))<\lambda_{2}(\mathcal{J}(\alpha))<\cdots
$$


be the sequence of all eigenvalues of $\mathcal{J}(\alpha)$ greater than $a+1$. The above reasoning and formula (4.7) prove

Theorem 6. The large $n$ asymptotic behaviour of $\lambda_{n}(\mathcal{J}(\alpha))$ is given by

$$
\lambda_{n}(\mathcal{J}(\alpha))=\sqrt{2} n^{\alpha}(1+\mathrm{O}(1)) .
$$

Open Problems. Two open problems about $\sigma_{\mathrm{p}}(\mathcal{J}(\alpha))$.

(i) We expect that there is no concentration of $\sigma_{\mathrm{p}}(\mathcal{J}(\alpha))$ to the right and left of $\sigma_{\mathrm{ess}}(\mathcal{J}(\alpha))$.

(ii) Is $\sigma_{\mathrm{p}}(\mathcal{J}(\alpha))$ in the "interior" gaps finite?

\section{Approximate Lengths of Bands ( $N$ Even)}

In this section we consider the Jacobi matrix $\mathcal{J}(\alpha)$ associated to a particular sequence $z_{1}, \ldots, z_{N}$ (all are powers $\varepsilon^{\gamma_{s}}$ of a small positive parameter $\varepsilon$ ) which will allow to compute the small $\varepsilon$ asymptotics of the lengths of the bands of $E$. This choice of the $z_{s}$ will also allow to give sufficient conditions (in terms of the above exponents $\gamma_{s}$ ) for $E$ being exactly the union of $N$ disjoint intervals.

Most of the proofs will be sketchy in order to avoid lengthy but straightforward calculations.

Definition $7\left(\mathcal{J}^{\varepsilon}(\alpha)\right)$. Let $\varepsilon$ be a positive number. We denote $\mathcal{J}^{\varepsilon}(\alpha)$ the Jacobi matrix $\mathcal{J}(\alpha)$ associated to a sequence $z_{1}=\varepsilon^{\gamma_{1}}, \ldots, z_{N}=\varepsilon^{\gamma_{N}}$ with $\gamma_{1}<\cdots<\gamma_{N}$

Lemma 8. Assuming that the sequence $\left\{\gamma_{k}\right\}$ is convex, the coefficients $c_{2 k}$ (see (3.1)) admit the following asymptotics, as $\varepsilon \rightarrow 0$ :

$$
c_{2 k} \sim-(-1)^{\frac{N}{2}-k}\left(z_{N} z_{N-1} \cdots z_{N-2 k+1}\right)^{-1} \Phi(k),
$$

where

$$
\Phi(k):=\frac{z_{N-2 k-1} z_{N-2 k-3} \ldots z_{1}}{z_{N-2 k} z_{N-2 k-2} \ldots z_{2}} .
$$

Proof. Indeed, the direct computation of

$$
\operatorname{tr}\left(\sum_{\substack{n_{1}, \ldots, n_{k} \\ n_{1}+\cdots+n_{k}=N-k}} X_{n_{1}} \ldots X_{n_{k}}\right)
$$

for a suitable choice of matrices $X_{i}$ shows that the dominant term among all components of the above traces is given by

$$
\operatorname{tr}\left(B_{N} B_{N-1} \cdots B_{N-2 k+1} A_{N-2 k} \cdots A_{2} A_{1}\right)
$$

for sufficiently small $\varepsilon$. This fact can be easily verified using the convexity of the sequence $\left\{\gamma_{s}\right\}$. Using our choice of $z_{s}=\varepsilon^{\gamma_{s}}$ and (5.2) we find

$$
c_{2 k} \sim \pm(-1)^{k} \varepsilon^{-\Delta_{k}}, \quad \text { as } \varepsilon \rightarrow 0,
$$


where

$$
\begin{aligned}
& \Delta_{k}:=\gamma_{N}+\gamma_{N-1}+\cdots+\gamma_{N-2 k+1} \\
& \quad+\left(\gamma_{N-2 k}-\gamma_{N-2 k-1}\right)+\cdots+\left(\gamma_{2}-\gamma_{1}\right) \text { for } k=1, \ldots, \frac{N}{2}, \\
& \Delta_{0}:=\gamma_{N}-\gamma_{N-1}+\gamma_{N-2}-\gamma_{N-3}+\cdots+\gamma_{2}-\gamma_{1},
\end{aligned}
$$

with $\gamma_{0}:=0$. Thus,

$$
\Delta_{s+1}-\Delta_{s}=2 \gamma_{N-2 s-1}, \quad s \geq 1 .
$$

Note that the $\Delta_{s}$ are positive and increasing. It follows that

$$
\Delta_{s+1}-\Delta_{s}=2 \gamma_{N-2 s-1}<2 \gamma_{N-2 s+1}=\Delta_{s}-\Delta_{s-1},
$$

i.e., the sequence $\left\{\Delta_{s}\right\}$ is concave.

Below we shall find approximate roots $\mu_{-s}$ (respectively $\mu_{+s}$ ) of the equation $P(\mu)=-2$ (respectively $P(\mu)=2), s=1, \ldots, N=2 M$. Since

$$
c_{2 M}=-\frac{2}{z_{N} z_{N-1} \ldots z_{1}}<0,
$$

it is clear that $P(\mu) \rightarrow-\infty$, as $\mu \rightarrow+\infty$, independently on the parity of $M$. Hence the largest root coincides with $\mu_{-1}$ and corresponds to the equation $P(\mu)=-2$.

It turns out that a rough approximation of the roots can be found by the following simple procedure. Although simple this rough approximation dramatically reduces the complexity of finding the roots and is sufficient for our aim. Surely this procedure works due to the special form of the coefficients of the polynomial $P$.

\section{First Step}

We look for the solutions of the following simplified equations (just keeping two leading terms of the polynomial $P$ ):

$$
c_{2 k} \mu^{k}+c_{2 k-2} \mu^{k-1}=0, \quad k=1, \ldots, \frac{N}{2} .
$$

The solutions of (5.7) are:

$$
\begin{aligned}
& \mu_{k} \equiv 0, \\
& \tilde{\mu}_{k}:=-\frac{c_{2 k-2}}{c_{2 k}} \sim \varepsilon^{\Delta_{k}-\Delta_{k-1}}, \text { as } \varepsilon \rightarrow 0 .
\end{aligned}
$$

Below (the second step) by using a better approximation of the polynomial $P$ we shall split the zero solution into smaller distinct roots.

Observe that all terms $c_{2 s} \tilde{\mu}_{k}^{s}$ decay, for $s \neq k-1, k$, faster than $c_{2 k} \tilde{\mu}_{k}^{k}$ and $c_{2 k-2} \tilde{\mu}_{k}^{k-1}$ as $\varepsilon \rightarrow 0$. Indeed, we have two possibilities:

- $s<k-1$. By concavity of $\left\{\Delta_{r}\right\}$ we have the following inequalities:

$$
\begin{gathered}
\Delta_{k}-\Delta_{s}=\Delta_{k}-\Delta_{k-1}+\cdots+\Delta_{s+1}-\Delta_{s} \\
>(k-s)\left(\Delta_{k}-\Delta_{k-1}\right) .
\end{gathered}
$$


But these inequalities are equivalent to

$$
s\left(\Delta_{k}-\Delta_{k-1}\right)-\Delta_{s}>k\left(\Delta_{k}-\Delta_{k-1}\right)-\Delta_{k}
$$

which by the form of $c_{2 s}$ implies faster decay of $c_{2 s} \tilde{\mu}_{k}^{s}$.

- $s>k$. Similarly we have

$$
\begin{gathered}
\Delta_{s-1}-\Delta_{k-1}=\Delta_{s-1}-\Delta_{s-2}+\cdots+\Delta_{k}-\Delta_{k-1} \\
<(s-k)\left(\Delta_{k}-\Delta_{k-1}\right) .
\end{gathered}
$$

Thus,

$$
\Delta_{s}-\Delta_{k}<\Delta_{s-1}-\Delta_{k-1}<(s-k)\left(\Delta_{k}-\Delta_{k-1}\right) .
$$

Again the last inequality is equivalent to

$$
k\left(\Delta_{k}-\Delta_{k-1}\right)-\Delta_{k}<s\left(\Delta_{k}-\Delta_{k-1}\right)-\Delta_{s}
$$

which implies the desired faster decay of $c_{2 s} \tilde{\mu}_{k}^{s}$.

\section{Second Step}

We assume that one can find "better" approximate roots by choosing

$$
\tilde{\mu}_{ \pm k}=\tilde{\mu}_{k}\left(1+\delta_{ \pm}(k)\right), \quad k=1, \ldots, \frac{N}{2}
$$

as the solutions of the "better" equation

$$
c_{2 k} \tilde{\mu}_{ \pm k}^{k}+c_{2 k-2} \mu_{ \pm k}^{k-1}= \pm 2
$$

(recall that we look for the solutions of $P(\mu)= \pm 2$ ).

Combining the asymptotic form for $c_{2 k}$ [see (5.3), (5.8) and (5.9)] we obtain

$$
\delta_{ \pm}(k) \sim \pm 2 \varepsilon^{\Delta_{k}-k\left(\Delta_{k}-\Delta_{k-1}\right)}
$$

as $\varepsilon \rightarrow 0$. Since $\tilde{\mu}_{+s} \sim \tilde{\mu}_{-s} \gg \tilde{\mu}_{+, s+1} \sim \tilde{\mu}_{-, s+1}$ as $\varepsilon \rightarrow 0$ we have

$$
\tilde{\mu}_{+s}-\tilde{\mu}_{-s}=\left(\delta_{+}(s)-\delta_{-}(s)\right) \tilde{\mu}_{s} \sim 4 \varepsilon^{\Delta_{s}-(s-1)\left(\Delta_{s}-\Delta_{s-1}\right)} .
$$

This allows to calculate the lengths of bands $\left(\sqrt{\tilde{\mu}_{+s}}, \sqrt{\tilde{\mu}_{-s}}\right)$ or $\left(\sqrt{\tilde{\mu}_{-s}}, \sqrt{\tilde{\mu}_{+s}}\right)$. In fact,

$$
\sqrt{\tilde{\mu}_{+s}}-\sqrt{\tilde{\mu}_{-s}}=\frac{\tilde{\mu}_{+s}-\tilde{\mu}_{-s}}{\sqrt{\tilde{\mu}_{+s}}+\sqrt{\tilde{\mu}_{-s}}} \sim 2 \varepsilon^{\Delta_{s}-\left(s-\frac{1}{2}\right)\left(\Delta_{s}-\Delta_{s-1}\right)}
$$

as $\varepsilon \rightarrow 0$.

The above arguments lead to

Theorem 9. Let $\mathcal{J}^{\varepsilon}(\alpha)$ be the Jacobi matrix defined by a convex sequence $\gamma_{1}<\cdots<\gamma_{N}$ as in Definition 7 . The lengths of the bands $\left(\sqrt{\tilde{\mu}_{-s}}, \sqrt{\tilde{\mu}_{+s}}\right)$ of the spectrum of $\mathcal{J}^{\varepsilon}(\alpha)$ lying in $\mathbb{R}_{+}$(the spectrum is symmetric w.r.t. zero) have the following asymptotics, as $\varepsilon \rightarrow 0$ :

$$
\sqrt{\tilde{\mu}_{+s}}-\sqrt{\tilde{\mu}_{-s}} \sim 2 \varepsilon^{\Delta_{s}-\left(s-\frac{1}{2}\right)\left(\Delta_{s}-\Delta_{s-1}\right)} .
$$


Remark. The above "approximate" lengths of the bands are decreasing as the parameter $s$ increases. Moreover, $\tilde{\mu}_{ \pm s}$ have different orders for different $s$. More precisely

$$
\tilde{\mu}_{-1}>\tilde{\mu}_{+1} \gg \tilde{\mu}_{+2}>\tilde{\mu}_{-2} \gg \ldots
$$

for $\varepsilon$ sufficiently small.

\section{Acknowledgements}

J. J. is supported in part by "INTAS" and in part by MSHE grant N N201 426533. S. N. is supported in part by "INTAS" and in part by RFBR grant 09-01-00515a. S. N. is also grateful to the Université Paris Diderot (where a part of this work has been done) for its hospitality.

Open Access. This article is distributed under the terms of the Creative Commons Attribution Noncommercial License which permits any noncommercial use, distribution, and reproduction in any medium, provided the original author(s) and source are credited.

\section{References}

[1] Berezanskiŭ, J. M.: Expansions in eigenfunctions of selfadjoint operators. In: Bolstein, R., Danskin, J.M., Rovnyak, J., Shulman, L. Translated from the Russian, Translations of Mathematical Monographs, vol. 17. American Mathematical Society, Providence (1968)

[2] Birman, M. S., Solomjak, M. Z.: Spectral Theory of Selfadjoint Operators in Hilbert Space. Mathematics and its Applications (Soviet Series). D. Reidel Publishing Co., Dordrecht (1987)

[3] Boutet de Monvel, A., Naboko, S., Silva, L. O.: The asymptotic behavior of eigenvalues of a modified Jaynes-Cummings model. Asymptot. Anal. 47(3-4), 291-315 (2006)

[4] Christiansen, J. S., Simon, B., Zinchenko, M.: Finite gap Jacobi matrices. I. The isospectral torus. Constr. Approx. 32, 1-65 (2010)

[5] Dombrowski, J., Pedersen, S.: Absolute continuity for unbounded Jacobi matrices with constant row sums. J. Math. Anal. Appl. 267(2), 695-713 (2002)

[6] Dombrowski, J., Pedersen, S.: Spectral transition parameters for a class of Jacobi matrices. Stud. Math. 152(3), 217-229 (2002)

[7] Janas, J., Malejki, M.: Alternative approaches to asymptotic behaviour of eigenvalues of some unbounded Jacobi matrices. J. Comput. Appl. Math. 200(1), 342-356 (2007)

[8] Janas, J., Moszyński, M.: Spectral properties of Jacobi matrices by asymptotic analysis. J. Approx. Theory 120(2), 309-336 (2003)

[9] Janas, J., Naboko, S.: Jacobi matrices with power-like weights-grouping in blocks approach. J. Funct. Anal. 166(2), 218-243 (1999)

[10] Janas, J., Naboko, S.: Spectral analysis of selfadjoint Jacobi matrices with periodically modulated entries. J. Funct. Anal. 191(2), 318-342 (2002) 
[11] Janas, J., Naboko, S.: Infinite Jacobi matrices with unbounded entries: asymptotics of eigenvalues and the transformation operator approach. SIAM J. Math. Anal. 36(2), 643-658 (2004)

[12] Janas, J., Naboko, S., Stolz, G.: Spectral theory for a class of periodically perturbed unbounded Jacobi matrices: elementary methods. J. Comput. Appl. Math. 171(1-2), 265-276 (2004)

[13] Janas, J., Naboko, S., Stolz, G.: Decay bounds on generalized eigenvalues of unbounded jacobi matrices and the singular spectrum. Int. Math. Res. Not. IMRN, pages Art. ID rnn144, 29 (2009)

[14] Khan, S., Pearson, D. B.: Subordinacy and spectral theory for infinite matrices. Helv. Phys. Acta 65(4), 505-527 (1992)

[15] Last, Y., Simon, B.: Eigenfunctions, transfer matrices, and absolutely continuous spectrum of one-dimensional Schrödinger operators. Invent. Math. 135(2), 329-367 (1999)

[16] Malejki, M.: Approximation of eigenvalues of some unbounded self-adjoint discrete Jacobi matrices by eigenvalues of finite submatrices. Opuscula Math. 27(1), 37-49 (2007)

[17] Moszyński, M.: Spectral properties of some Jacobi matrices with double weights. J. Math. Anal. Appl. 280(2), 400-412 (2003)

[18] Pedersen, S.: Absolutely continuous Jacobi operators. Proc. Am. Math. Soc. 130(8), 2369-2376 (2002)

[19] Silva, L. O.: Uniform and smooth Benzaid-Lutz type theorems and applications to Jacobi matrices. Operator theory, analysis and mathematical physics. Oper. Theory Adv. Appl., vol. 174, 173-186. Birkhäuser, Basel (2007)

[20] Silva, L. O.: On the spectral analysis of selfadjoint Jacobi matrices and its applications. PhD thesis. St-Petersburg State University, Russia (2002)

[21] Simonov, S.: An example of spectral phase transition phenomenon in a class of Jacobi matrices with periodically modulated weights. Operator theory, analysis and mathematical physics. Oper. Theory Adv. Appl., vol. 174, 187-203. Birkhäuser, Basel (2007)

[22] Sodin, M., Yuditskii, P.: Almost periodic Jacobi matrices with homogeneous spectrum, infinite-dimensional Jacobi inversion, and Hardy spaces of character-automorphic functions. J. Geom. Anal. 7(3), 387-435 (1997)

[23] Stone, M. H.: Linear transformations in Hilbert space. American Mathematical Society Colloquium Publications, vol. 15. American Mathematical Society, Providence (1990)

[24] Teschl, G.: Jacobi Operators and Completely Integrable Nonlinear Lattices. Mathematical Surveys and Monographs, vol. 72. American Mathematical Society, Providence (2000)

[25] Zielinski, L.: Eigenvalue asymptotics for a class of Jacobi matrices. In: Hot Topics in Operator Theory: Conference Proceedings, Timişoara, June 290-July 4, 2006. Theta Ser. Adv. Math., vol. 9, pp. 217-229. Theta, Bucharest (2008) 
Anne Boutet de Monvel

Institut de Mathématiques de Jussieu

Université Paris Diderot Paris 7

175 rue du Chevaleret

75013 Paris

France

e-mail: aboutet@math.jussieu.fr

Jan Janas $(\bowtie)$

Institute of Mathematics

Polish Academy of Sciences

ul. Św. Tomasza 30

31-027 Kraków

Poland

e-mail: najanas@cyf-kr.edu.pl

Serguei Naboko

Department of Mathematical Physics,

Institute of Physics

St. Petersburg University

Ulianovskaia 1

198904, St. Petergoff, St. Petersburg

Russia

e-mail: naboko@snoopy ·phys.spbu.ru

Received: June 27, 2009.

Revised: October 26, 2010. 